\title{
The Fabry-Perot interferometer for the DELPHI Ring Imaging Cherenkov detector
}

\author{
E. Fokitis et al
}

\begin{abstract}
The performance of a refractometer for operation in the DELPHI Ring Imaging Cherenkov detectors for operation in the far UV region is presented. Comparisons are made with performance in standard laboratory conditions. Data on absolute refractivities are given for reference samples of air, argon, Freon 12 as well as on gaseous $C_{4} F_{10}$ from the Forward RICH detector of DELPHI. The accuracy of the determination of the $\mathrm{n}-1$ using this refractometer at the $253.7 \mathrm{~nm}$ is estimated about $1 \%$ for $C_{4} F_{10}$.
\end{abstract}

Contribution to the Como conference "Advanced technology and Particle Physics", Como, Italy, 3-7 October 1994. 


\title{
The Fabry-Perot Interferometer for the DELPHI Ring Imaging Cherenkov Detector
}

\author{
E. Fokitis, S. Maltezos, K. Patrinos, M. Dris, H. Rahmani and P. Papandreopoulos
}

Physics Department, National Technical University of Athens,

Zografou Campus, 15780 Athens, Greece

The performance of a refractometer for operation in the DELPHI Ring Imaging Cherenkov detectors for operation in the far UV region is presented. Comparisons are made with performance in standard laboratory conditions. Data on absolute refractivities are given for reference samples of air, argon, Freon 12 as well as on gaseous C4F10 from the Forward RICH detector of DELPHI. The accuracy of the determination of the n-1 using this refractometer at the $253.7 \mathrm{~nm}$ is estimated about $1 \%$ for $C_{4} F_{10}$.

\section{INTRODUCTION}

The performance of the Ring Imaging Cherenkov (RICH) detectors, using gas radiators can be monitored more reliably if the value of the refractive index of the radiator is measured frequently in the wavelength range in which the RICH detector is sensitive. For gaseous RICH detectors the Fabry-Perot [1], [2] pressure tunable UV refractometer presents an excellent possibility for continuous automatic monitoring of refractivities down into the VUV region.

The data to be presented here are based on a refractometer prototype appropriate for operation in the UV region.

The advantage of the Fabry-Perot type refractometer is that it can operate in a wide spectral range. The obtained accuracy can be at the level of better than a few per thousands even at the far ultraviolet region.

We will, in section 2, describe the construction and installation of the refractometer in the DELPHI [3] experiment. We will also report on the performance with reference samples, in the section 3. In the fourth section, we will present results of measurements of refractivities reaching the far ultraviolet region $(253.7 \mathrm{~nm})$ at the Forward RICH Detector of DELPHI.

1.1. The motivation for refractivity measurement in RICH detectors.

A charged particle traversing a dielectric medium with a velocity greater than $\mathrm{c} / \mathrm{n}$, where $\mathbf{n}$ is the refractive index of the medium, emits
Cherenkov radiation at an angle $\theta_{C}$ with respec$\mathrm{t}$ to the direction of the particle. This angle is given by :

$\cos \theta_{c}=\frac{1}{\beta n}$

$\beta$ is the ratio of the particle velocity to the speed of light in vacuum. One can select a sample of charged particles with $\beta$ approaching 1 . Then from the mean Cherenkov angle one can determine directly a value for the refractivity $n-1$, averaged over the wavelength window of the detector.

This method needs a full analysis of data from a ring imaging detector and tracking chambers and has several uncertainties. It is better to have a direct refractivity measurement for the gas radiator.

\subsection{Principle of Operation of the Refractometer.}

When light from an extended monochromatic source, with intensity $\mathrm{I}_{o}$, is transmitted through a pair of semitransparent mirrors (etalon) (see Fig.1) the total intensity $I_{t o t}$ recorded at the focal plane of the lens $L_{2}$ is determined by multiple interference between the mirrors. It is given by [2] :

$$
I_{\text {tot }}=\frac{I_{0}[T /(1-R)]^{2}}{1+\mathcal{F} \cdot \sin ^{2}\left(\frac{2 \pi d \cos \theta}{\lambda} \cdot n\right)}
$$

where $\mathcal{F} \equiv 4 R /(1-R)^{2}, \mathrm{R}$ the reflectivity, A the absorbance of the mirrors, $T=1-R-A$ and $n$ 


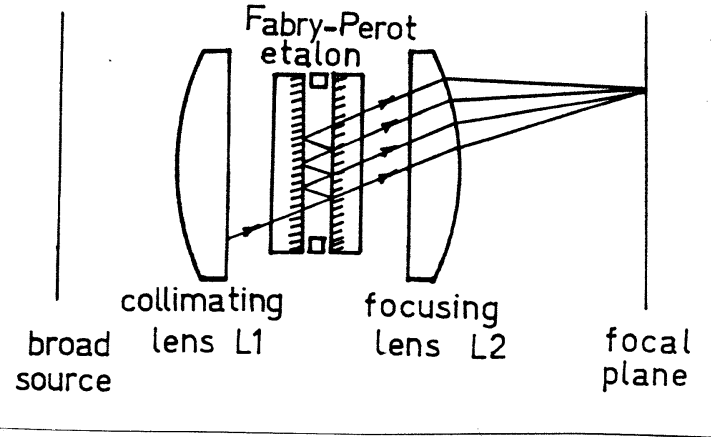

Figure 1. Principle of the Fabry-Perot interferometer.

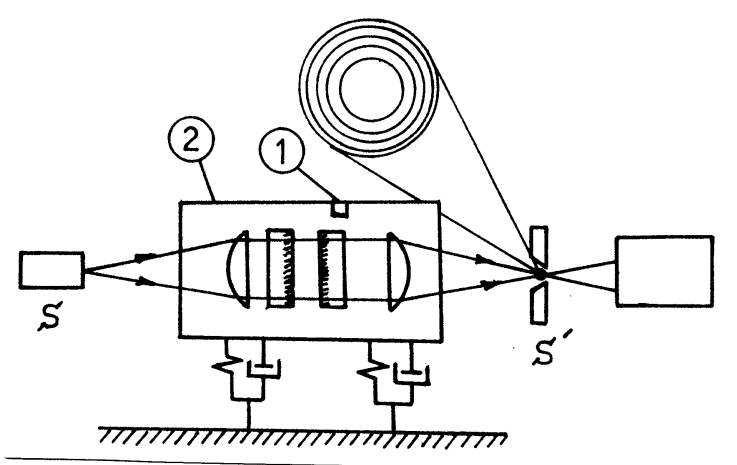

Figure 2. Schematics of optics and mechanical structure of Refractometer.

is the refractive index of the sample between the etalon mirrors with distance $d$. As the pressure read by the pressure sensor (1) inside the chamber (2) in figure 2 gradually increases during the introduction of the gas sample, the interference fringe pattern at the focal plane $S^{\prime}$ of the exit lens changes according to equation (2), since $\mathbf{n}$ depends on the gas pressure [1], [6]. This variation is recorded using a photomultiplier tube (3) wich samples a the fraction of the central fringe passing through the pinhole $S^{\prime}$.

The determination of the refractive index for the sample is done by counting the number of interference fringes $N(P)$ from vacuum up to a final pressure $(\mathrm{P})$ and then applying the relationship

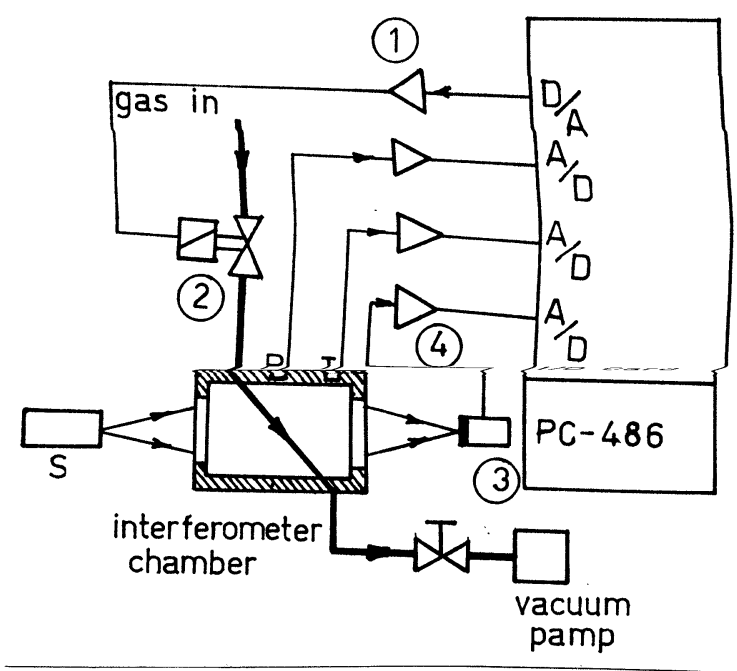

Figure 3. Schematics of monitoring and control of the refractometer.

$n(P)-1=\frac{\lambda}{2 d} \cdot N(P)$.

\section{INSTALLATION OF THE REFRA- CTOMETER AT DELPHI}

The basic requirements are operation down to the VUV region, flexibility of optical construction to allow mirror parallelism with an accuracy of the order of $\lambda / 20$, automatic control of the sample insertion into the chamber containing the etalon and online data acquisition. The mirror parallelism is achieved by a set of three equal thickness spacers glued between the etalon mirrors. The stability of the parallelism and of the other optical parts of the interferometer is achieved by a specially designed optical table.

The schematics of automatic monitoring and gas sample flow control are seen in figure 3 .

A PC-486 computer equipped with an I/O card with $A / D$ converters for monitoring of sensors and $\mathrm{D} / \mathrm{A}$ converters for flow control of the gas insertion. The signal from $\mathrm{D} / \mathrm{A}$ amplified by a current amplifier (1), drives the analog control valve (2) in order to achieve a linear dependence of absolute pressure versus time.

Absolute pressure, light intensity and temperature averaged every 400 succesive samplings are 


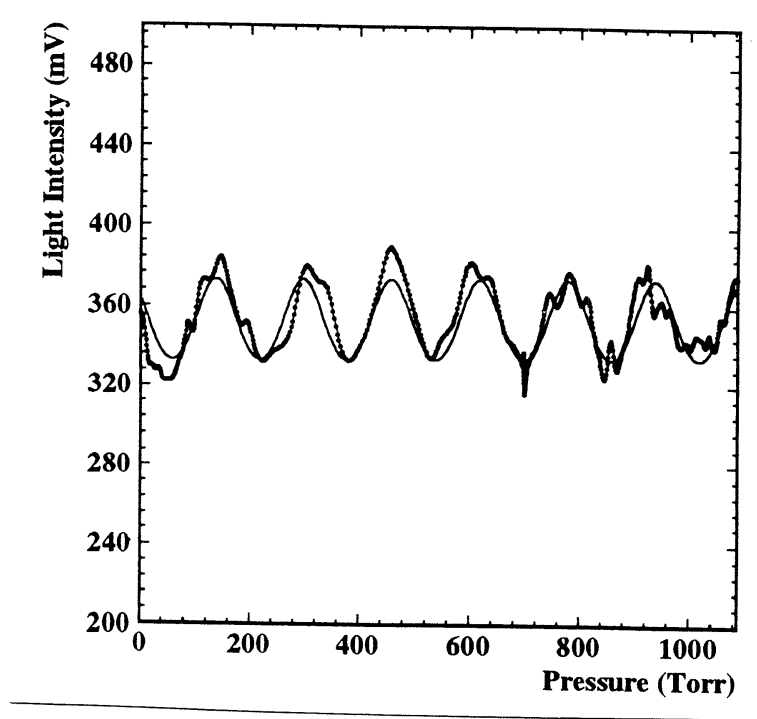

Figure 4. Interferogram for argon sample using $\mathrm{Hg}-\mathrm{Ne}$ low pressure lamp with filter at $253.7 \mathrm{~nm}$.

recorded.

The photomultiplier used was a Hammamatsu 931B with $\mathrm{p}$-Terpenyl wavelength shifter (3). Its signal is amplified by a low noise two stage operational amplifier (4).

\section{REFRACTOMETER PERFORMANCE USING REFERENCE GASES}

The quality of the on-line and control system developed for the DELPHI refractometer was tested using two different etalons made of fused silica, one with spacer thickness $2.95 \mathrm{~mm}$ and 35 $\mathrm{mm}$ mirror diameter and the other with thickness $2.13 \mathrm{~mm}$ and $25 \mathrm{~mm}$ mirror diameter.

An example of interferogram appears in figure 4 using argon as a reference sample. The light source used was a Hg-Ne pen-ray natural mercury lamp. The wavelength of $253.7 \mathrm{~nm}$ was selected with the help of an interference filter [1]. It can be shown that the refractivity can be obtained by a fitting procedure on the data of such interferograms. The fitted curve is the following modified Airy function:

$$
I(p)=a_{4}+a_{6} p+\frac{a_{3}}{\left[1+a_{5} \sin ^{2}\left(a_{1}+\frac{2 \pi d}{\lambda} a_{2} p^{a_{7}}\right)\right]}(3)
$$

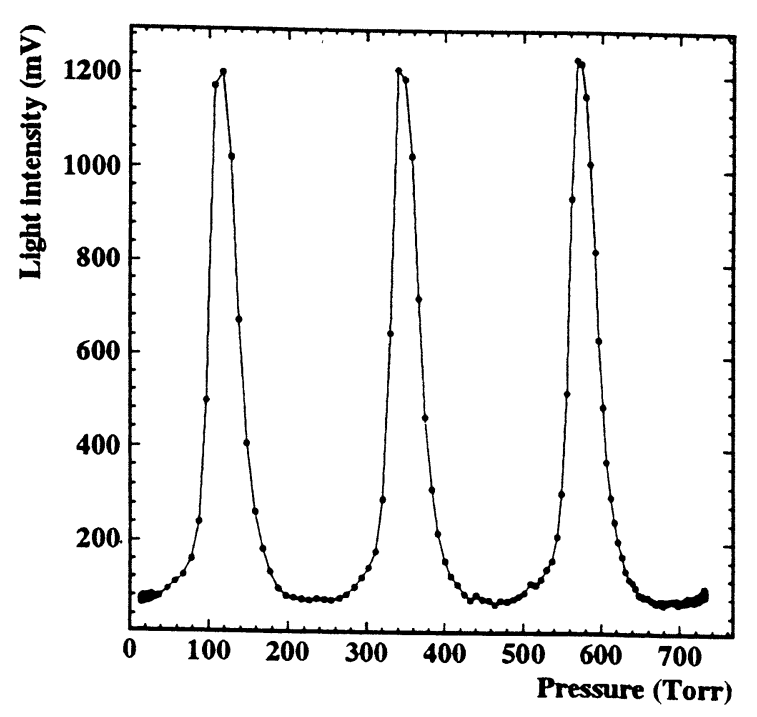

Figure 5. Interferogram of He-Ne laser at 632.8n$\mathrm{m}$ using etalon with spacer $3 \mathrm{~mm}$ and sample atmospheric air.

where $\mathrm{I}(\mathrm{p})$ is the light intensity signal, $\mathrm{p}$ the pressure and $\mathrm{a}_{i}, \mathrm{i}=1$ to 7 the fit parameters.

A mean refractivity of argon, based on the results of the fits of 5 different interferograms, for $\lambda=253.7 \mathrm{~nm}$, at pressure 760 Torr and temperature $0{ }^{\circ} C$, is $306.5 \times 10^{-6} \pm 1.5 \%$. This is consistent with the reference [4] (See Table 1 at section 4). We obtain for 1088 Torr a refractivity of $407 \times 10^{-6} \pm 1.5 \%$ at $23{ }^{\circ} \mathrm{C}$. There are no published values for such pressure at this wavelength for argon.

Further tests, on laboratory environment, with the first etalon and laser source at $632.8 \mathrm{~nm}$ gave for the atmospheric air sample, the interferogram seen in figure 5 .

We observe, as expected, a much cleaner peak structure (finesse) than in the case of the natural mercury interferogram of figure 4 . The lower finesse of the spectral lamp is due mainly to the presence of several isotopes in the natural $\mathrm{Hg}$ and to the stronger effect of the mirror defects in the wavelengths of the far UV. The good fit when using laser source indicates that our set-up has adequate protection from mechanical vibrations and reasonable linearity in the pressure measurement. 


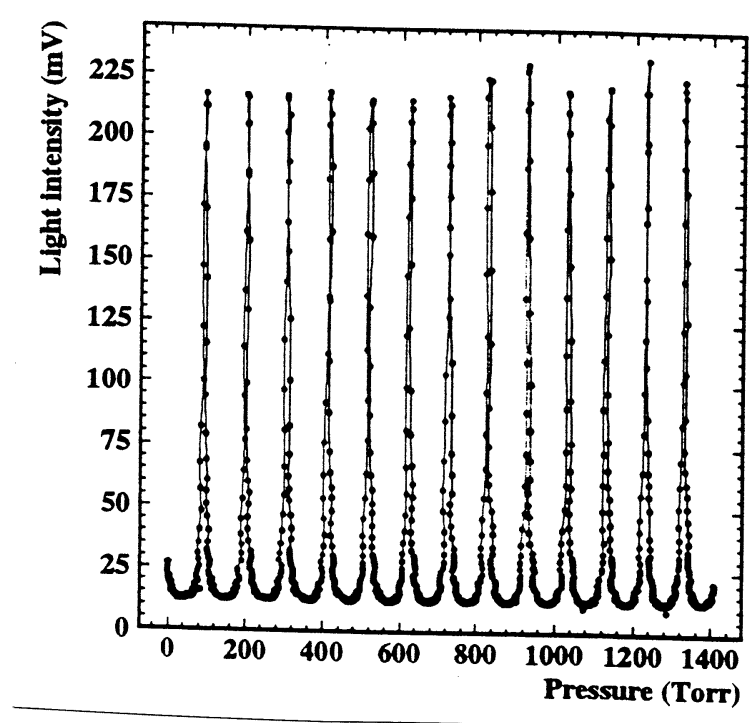

Figure 6. Interferogram with a $\mathrm{He}-\mathrm{Ne}$ laser at $543.5 \mathrm{~nm}$ for a sample of Freon 12.

The accuracy of the pressure sensor is $\pm 0.2 \%$ at full scale, while the photomultiplier signal had a signal to noise ratio of the order of 50-100 at 1200 volt depending on the intensity of the spectral line selected for the refractivity measurement.

\subsection{Performance with gases of greater re- fractivity.}

The possibility to use this type of refractometer for heavier compounds is shown by taking an interferogram for a gaseous fluorocarbon such as Freon 12 , in laboratory conditions, at wavelength $543 \mathrm{~nm}$, see figure 6 .

There is a tendency for the width of each peak in the pressure axis to increase with pressure as it is expected for a non ideal gas. By using the compressibility factors of this non-ideal gas from literature, and by fitting our interferometric data one can in principle obtain usefull information.

\section{ONLINE REFRACTIVITY MEASUREMENT OF THE FORWARD RICH GAS RADIATOR}

The in situ measurement of the $C_{4} F_{10}$ sample of the Forward RICH gas radiator is carried out after a proper purge of the piping of $15 \mathrm{~m}$

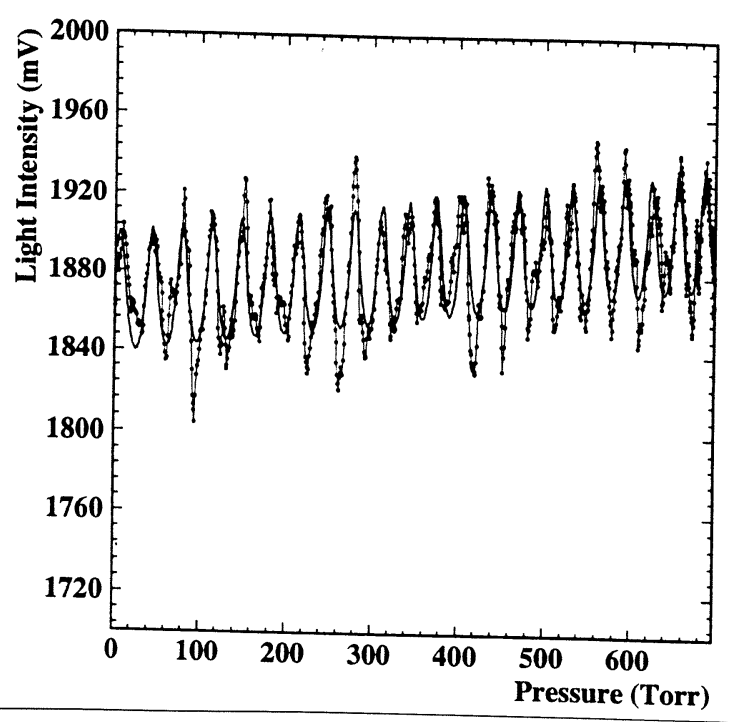

Figure 7. Interferogram at DELPHI site with sample from the Forward RICH detector at wavelength $253.7 \mathrm{~nm}$.

length, connecting the refractometer with the detector [7]. Figure 7 shows an interferogram for the $C_{4} F_{10}$ Cherenkov gas radiator. The data are fitted again to the fumction described with equation (3). We extract directly the refractive index at the final pressure of 700 Torr.

Figure 8 compiles the average values of refractivity obtained for the Forward RICH detector and the preliminary value obtained from the offline analysis of saturated tracks ( $\beta$ approaching 1) for the same sample at the same time. The refractive index obtained from the analyses of ring images was scaled to the corresponding $23{ }^{\circ} \mathrm{C}$. The dashed curve is a fit of the DELPHI data points with a empirical function of the form :

$n-1=a+B \cdot \frac{1}{\lambda_{0}^{-2}-\lambda^{-2}}$

as sugested by Abjean et al [6].

We also show some results from measurements of refractivity from Seguinot and Ypsilantis [8] for liquid phase and extrapolated to gaseous phase using the Lorentz -Lorenz equation. We observe that the measurements of the $n-1$ with the refractometer at $253.7 \mathrm{~nm}$ can be extrapolated rather 


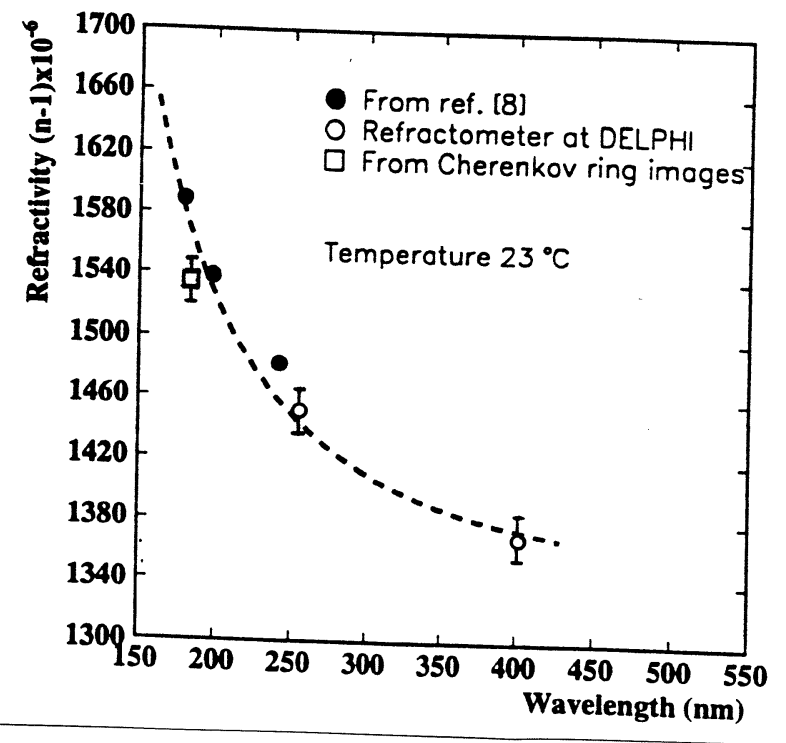

Figure 8. Dispersion of refractivity of $C_{4} F_{10}$ from visible to vacuum $\mathrm{UV}$ range.

succesfully to the range around $185 \mathrm{~nm}$ if a similar parametrization is used as for the Seguinot data.

These results suggest the usefullness of measurements of refractivity at higher wavelengths since an extrapolation at lower wavelengths can be done succesfully.

We summarize our results in Table 1 by presenting refractivities obtained in laboratory and at DELPHI for various samples. Literature values are also shown when available [4],[5].

\section{CONCLUSIONS}

We have installed and tested in the DELPHI Farward RICH detector a UV refractometer based on a Fabry-Perot pressure tunable interferometer. The conclusions are :

-Interferograms of gaseous $\mathrm{C}_{4} \mathrm{~F}_{10}$ indicate very stable interference patterns. The sensitivity is of the order of $1 / 10$ of a fringe down to the far UV range.

- The sensitivity of the interferometer allows for reliable measurements of refractivity versus wavelength ( dispersion).

- The duration of data taking for a sufficiently precise value of $n-1$ is of the order of a few

\begin{tabular}{|c|c|c|c|c|c|c|}
\hline \multicolumn{2}{|c|}{ SAMPLE } & ATM. AIR & ARGON & FREON 22 & FREON 12 & $\mathrm{C}_{4} \mathrm{~F}_{10}$ \\
\hline \multirow{2}{*}{$\begin{array}{l}\text { W.L. } \\
\text { (nm) }\end{array}$} & $\mathrm{T}$ & $0{ }^{\circ} \mathrm{C}$ & $0^{\circ} \mathrm{C}$ & $20{ }^{\circ} \mathrm{C}$ & $20{ }^{\circ} \mathrm{C}$ & $23{ }^{\circ} \mathrm{C}$ \\
\cline { 2 - 7 } & $\mathrm{P}$ & $760 \mathrm{Torr}$ & $760 \mathrm{Torr}$ & $760 \mathrm{Torr}$ & 760 Torr & $760 \mathrm{Torr}$ \\
\hline 253.7 & $\mathrm{~A}$ & $323 \pm 0.4 \%$ & $307 \pm 0.4 \%$ & - & - & $1450 \pm 0.8 \%$ \\
& $\mathrm{~B}$ & 317.0 & 303.6 & - & - & - \\
\hline 404.7 & $\mathrm{~A}$ & $293 \pm 0.7 \%$ & $291 \pm 0.7 \%$ & - & - & $1370 \pm 1.2 \%$ \\
& $\mathrm{~B}$ & 297.8 & 285.6 & - & - & - \\
\hline 543.5 & $\mathrm{C}$ & $296 \pm 1.1 \%$ & - & $760 \pm 0.2 \%$ & $1070 \pm 0.5 \%$ & - \\
\hline 632.8 & $\mathrm{C}$ & - & - & $758 \pm 0.7 \%$ & $1066 \pm 1.0 \%$ & - \\
\hline
\end{tabular}

A : DELPHI Refractometer measurements, etalon $2.13 \mathrm{~mm}$ B : Literature values

$C$ : Lab. measurements with etalons $0.975 \mathrm{~mm}$ and $3.00 \mathrm{~mm}$

Table 1

Summary of Results of interferometric measurements at DELPHI and in laboratory.

minutes.

\subsection{Prospects}

(1) We aim at measuring $n-1$ at lower wavelengths,$\lambda<200 \mathrm{~nm}$ by improving the optoelectronic chain so that adequate sensitivity for fringe detection is obtained at this wavelength range.

(2) The refractometer will also be connected with the $C_{5} F_{12}$ system of the Barrel RICH detector.

(3) We are in the process of exploiting data obtained with this refractometer in comparison with results for the same running period from analyses of Cherenkov ring images, to improve the quality of particle identification.

\section{Acknowledgements}

We thank our DELPHI collaborators and in particular M. Davenport, A. Filippas, G.Lenzen, E. Rosso and O. Ullaland for continuous support throughout the development of this project. We also wish to thank G. Read of Clarendon Laboratory and A. Braem of CERN Optics Lab for excellent work in optical componennts.

\section{REFERENCES}

1. Interferometric applications in High Energy Physics Experiments (Nucl. Phys. B,Vol. 23A, P.433 Supplement, 1991) 
2. The Fabry-Perot interfrometer J.M. Vaughan 1st. Edition.

3. The DELPHI detector at LEP, NIM A303(191) 233

4. ENCYCLOPEDIE DES GAZ - L'AIR LIQUIDE

5. Tamosaitis et al. ANL Report No.6916, 1964 $\&$ Landolf and Bornstein.

6. Abjean et al, NIM, A292(1990)593

7. G. Lenzen et al. NIM A343(1994)268-272.

8. Seguinot, CERN-EP/89-92, LPC/89-25 (1989) 